\title{
Prolonged transesophageal echocardiography during percutaneous closure of the left atrial appendage without general anesthesia: the utility of the Janus mask Échocardiographie transosophagienne prolongée pendant la
fermeture percutanée de l'appendice auriculaire gauche sans
anesthésie générale: l'utilité du masque de Janus
}

\author{
Alberto Zangrillo, MD • Patrizio Mazzone, MD • Carmine D. Votta, MD • \\ Nicola Villari, MD • Paolo Della Bella, MD • Fabrizio Monaco, MD \\ Received: 24 February 2016/Revised: 6 April 2016/Accepted: 19 April 2016/Published online: 26 April 2016 \\ (c) Canadian Anesthesiologists' Society 2016
}

\begin{abstract}
Purpose Left atrial appendage (LAA) closure is an interventional procedure increasingly used to prevent stroke in patients with permanent atrial fibrillation and contraindications to anticoagulation therapy. As this procedure requires a relatively immobile patient and performance of continuous and prolonged transesophageal echocardiography (TEE), it is usually performed under general anesthesia. In this case series, we describe the feasibility of prolonged TEE for percutaneous LAA closure using a new noninvasive ventilation device that can avoid the need for endotracheal intubation and general anesthesia.
\end{abstract}

Clinical features Percutaneous LAA closure was performed under deep sedation in three elderly patients

Electronic supplementary material The online version of this article (doi:10.1007/s12630-016-0659-1) contains supplementary material, which is available to authorized users.

A. Zangrillo, MD · C. D. Votta, MD (凶) ·

N. Villari, MD · F. Monaco, MD

Department of Anesthesia and Intensive Care, IRCCS San Raffaele Scientific Institute, Via Olgettina 60, 20132 Milan, Italy

e-mail: carmine.d.votta@virgilio.it

A. Zangrillo, MD

Vita-Salute San Raffaele University, Milan, Italy

P. Mazzone, MD · P. Della Bella, MD

Arrhythmology and Electrophysiology Unit, Cardio-Thoracic-

Vascular Department, IRCCS San Raffaele Scientific Institute,

Milan, Italy with permanent atrial fibrillation. Sedation was obtained with a combination of midazolam, propofol, and remifentanil. Continuous intraoperative TEE was performed through the port of the newly available Janus mask (Biomedical Srl; Florence, Italy), allowing for noninvasive ventilation (pressure support $=12-16 \mathrm{~cm} \mathrm{H}_{2} \mathrm{O}$; positive end-expiratory pressure $=7 \mathrm{~cm} \mathrm{H}_{2} \mathrm{O} ; \mathrm{FIO}_{2}=0.3$ ) in these spontaneously breathing patients. The total procedure times ranged from 75 $90 \mathrm{~min}$. The patients reported excellent satisfaction with the sedation received in terms of discomfort experienced during the procedure, capacity to recall the procedure, and comfort with the mask. The operators also rated the procedural conditions as excellent.

Conclusion Deep sedation with noninvasive ventilation may be a reasonable and safe alternative to general endotracheal anesthesia in patients requiring prolonged TEE for noninvasive cardiac procedures, including LAA closure.

Résumé

Objectif La fermeture de l'appendice auriculaire gauche $(A A G)$ est une procédure interventionnelle de plus en plus utilisée pour prévenir les accidents vasculaires cérébraux chez les patients souffrant de fibrillation auriculaire chronique ou présentant des contre-indications à l'anticoagulothérapie. Comme cette intervention requiert que le patient soit relativement immobile et une échocardiographie transœesophagienne (ETO) continue et prolongée, elle est habituellement réalisée sous anesthésie générale. Dans cette série de cas, nous décrivons la faisabilité d'une ETO prolongée pour la fermeture 
percutanée de l'AAG à l'aide d'un nouveau dispositif de ventilation non invasif qui permet d'éviter l'intubation endotrachéale et l'anesthésie générale.

Éléments cliniques La fermeture percutanée de l'AAG a été réalisée sous sédation profonde chez trois patients âgés atteints de fibrillation auriculaire chronique. La sédation a été obtenue avec un mélange de midazolam, de propofol et de rémifentanil. Une ETO peropératoire continue a été réalisée via le port du masque de Janus (Biomedical Srl; Florence, Italie) nouvellement commercialisé, qui a permis une ventilation non invasive (aide inspiratoire $=12-16 \mathrm{~cm}$ $\mathrm{H}_{2} \mathrm{O}$; pression positive télé-expiratoire $=7 \mathrm{~cm} \mathrm{H}_{2} \mathrm{O} ; \mathrm{FiO}_{2}$ $=0,3)$ de ces patients respirant spontanément. Les temps totaux pour l'intervention s'étalaient entre 75-90 min. Les patients ont rapporté être très satisfaits de la sédation reçue en termes d'inconfort ressenti pendant l'intervention, de leur capacité à se souvenir de l'intervention, et du confort du masque. Les opérateurs ont également évalué les conditions procédurales comme étant excellentes.

Conclusion Une sédation profonde accompagnée d'une ventilation non invasive pourrait constituer une alternative raisonnable et sécuritaire à l'anesthésie générale avec intubation endotrachéale chez les patients nécessitant une ETO prolongée pour des interventions cardiaques non invasives, notamment la fermeture de l'AAG.

Percutaneous closure of the left atrial appendage (LAA) is a procedure increasingly performed in patients with atrial fibrillation at high risk of stroke and with contraindications to long-term oral anticoagulation therapy. ${ }^{1}$ The procedure involves transatrial septal puncture, angiography of the left appendage, and positioning of an occluding device. The patient must be immobile during all these phases, as a sudden movement can increase the risk of significant complications, including atrial perforation and pericardial tamponade. ${ }^{2}$ To keep patients immobile and allow a prolonged three-dimensional transesophageal echocardiography (3D-TEE) examination, percutaneous LAA is usually performed under general anesthesia.

The risk of general anesthesia in the elderly is associated not only with challenges of airway control, potential for residual neuromuscular block, and an increased risk of perioperative pulmonary complications ${ }^{3,4}$ but also with the risk of cognitive decline. ${ }^{5}$ Furthermore, general anesthesia may increase costs and reduce the number of procedures that can be performed daily. ${ }^{6}$

Continuous TEE is an essential tool to help the interventional cardiologist perform the procedure, and it allows for the administration of lower doses of contrast medium and an overall lower exposure to radiation for the patient and the operating team. Nevertheless, it can be challenging for the patient to tolerate what can be a protracted TEE examination.

Herein, we present a case series of three patients who underwent percutaneous LAA closure under continuous TEE and deep sedation. The procedure was facilitated with the use of a new noninvasive ventilation mask that can be placed around the TEE probe.

\section{Case series}

With San Raffaele Scientific Institute Ethical Committee approval (Milan; protocol no. VP/50ER/mm) and patients' written consent, three elderly patients $(80,76$, and $74 \mathrm{yr})$ underwent percutaneous LAA closure under continuous 3DTEE guidance (Vivid E9; GE Medical Systems, Milwaukee, WI, USA). Monitoring included electrocardiography, invasive blood pressure monitoring, capnography, and pulse oximetry. Sedation was performed with midazolam $(2 \mathrm{mg})$, continuous infusions of propofol $\left(40-60 \mathrm{mg} \cdot \mathrm{hr}^{-1}\right.$ and remifentanil $\left(0.025-0.05 \mu \mathrm{g} \cdot \mathrm{kg}^{-1} \cdot \mathrm{min}^{-1}\right)$. Each patient's sedation level was evaluated by means of the Observer's Assessment of Alertness/Sedation (OAA/S) scale ${ }^{7}$ which
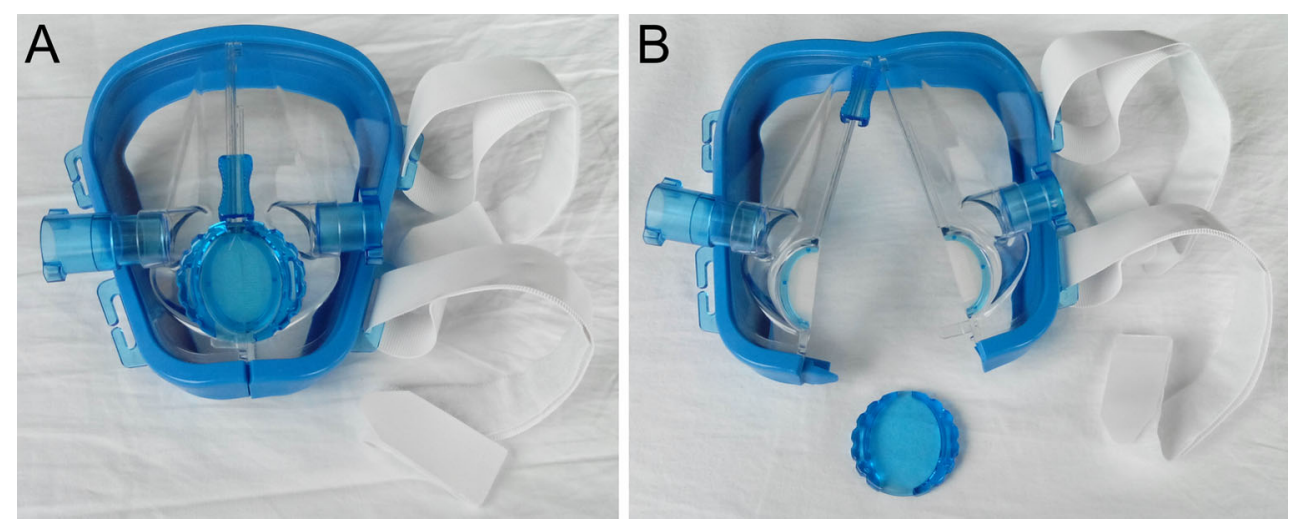

Fig. 1 The Janus mask for noninvasive ventilation in its closed (A) and open (B) appearance 

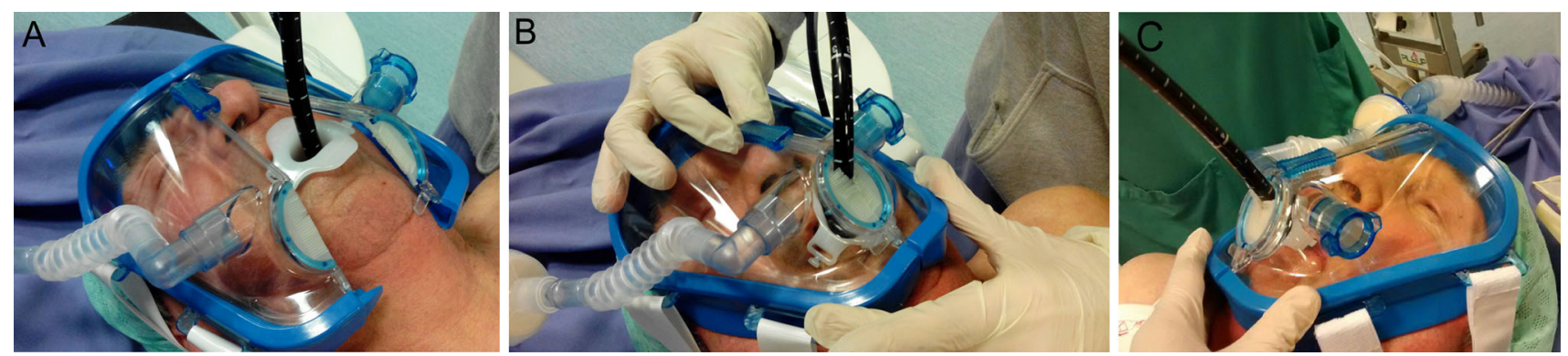

Fig. 2 The Janus mask: open on a patient's face with a transesophageal echocardiography probe inserted in his mouth (A), during its closure (B), and closed around the probe (C)

ranges from $0-5$, where $0=$ the patient does not respond to squeezing the trapezius, and $5=$ the patient responds readily to name spoken in normal tone. Once the patient's OAA/S score was 1 , the TEE probe was inserted. Thereafter, the Janus mask (Biomedical Srl; Florence, Italy) (Fig. 1A and B) was placed on the patient's face around the probe without the necessity of removing the device. ${ }^{8}$ The patient's spontaneous ventilation was assisted with pressure support of 12-16 cm $\mathrm{H}_{2} \mathrm{O}$ and a positive end-expiratory pressure of $7 \mathrm{~cm} \mathrm{H}_{2} \mathrm{O}$ with an $\mathrm{F}_{1} \mathrm{O}_{2}=0.3$. Oxygen saturation during the procedures was 94-98\%, and arterial partial pressure of carbon dioxide ranged from $34.0-44.9 \mathrm{mmHg}$.

The procedures lasted 75-90 min and were uneventful, and the operators rated the procedural conditions as excellent (5 on a scale of 1-5). The OAA/S scale remained at 1 throughout the procedures and reached 5 from one to three minutes after sedation was discontinued. Patients tolerated the mask favourably and did not recall the procedure. Furthermore, all patients assessed their pain at zero on the numeric rating scale both during and after the procedure. One of the three echocardiography teams complained of excessive image attrition due to difficulties in moving the TEE probe. In particular, they experienced difficulty in introducing the TEE probe into the patient's mouth due to suboptimal gliding of the probe through the flexible sheets of the mask's central port, which are designed to limit airway leakage around the probe.

\section{Discussion}

We present a small case series of patients undergoing LAA closure with continuous 3D-TEE performed without general anesthesia. To perform such an interventional procedure, the patient must remain immobile throughout the entire procedure. ${ }^{2}$ For this reason, as well as the need for continuous and prolonged TEE, LAA closure is mainly performed under general anesthesia, which itself is burdened by an increased risk of pulmonary complications. $^{4,9}$ The possibility of substituting general anesthesia with sedation while keeping the patient

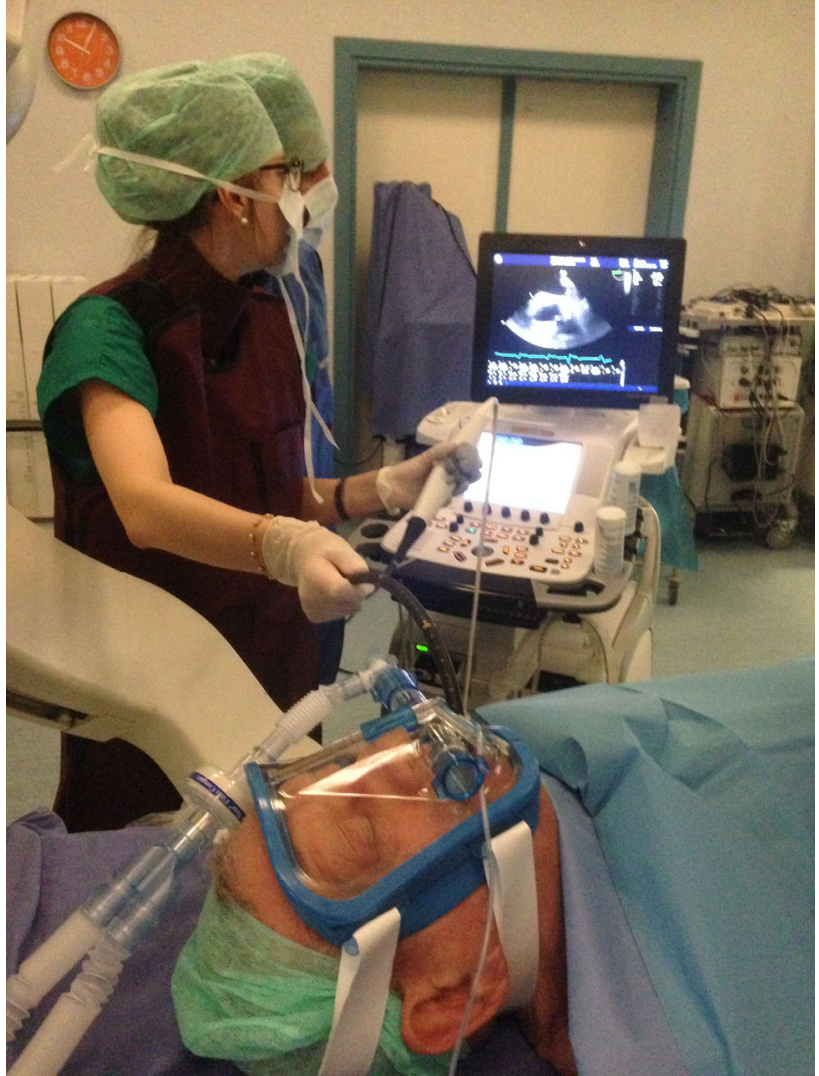

Fig. 3 Transesophageal echocardiography performed during noninvasive ventilation

spontaneously breathing is debatable. Deep sedation is associated with an increased risk of upper airway collapse, ${ }^{10}$ and assisting the patient's breathing with is recommended. The Janus mask has several advantages. For example, the mask can be placed on the patient's face and tested before induction of sedation as a normal mask for non-invasive ventilation. The mask has a hole that allows TEE examination during non-invasive ventilation, and it can be opened (and closed) around the endoscopic probe, facilitating insertion of the TEE probe into the patient's mouth (video; available as Electronic Supplementary Material). The mask can also be used in an emergency or 
unplanned situation (even if this was not the case in our case series) (Fig. 2A, B, and C, Fig. 3).

The possibility to perform LAA closure without general anesthesia has many advantages; for example, it allows less operating room utilization and obviates the potential side effects of general anesthesia, particularly in the elderly. It is reasonable to anticipate that cardiology (e.g., transfemoral aortic valve implantation and mitral valve repair), gastroenterology, and thoracic/pulmonology ${ }^{11}$ procedures could be managed in a similar way to avoid general anesthesia. In cardiology procedures, this would minimize the administration of contrast medium.

Funding Departmental funds only.

Conflicts of interest None declared.

Author contributions Alberto Zangrillo conceived the report, read the draft, and contributed to the final version of the manuscript. Patrizio Mazzone and Paolo Della Bella, the two electrophysiologists, performed the interventional procedure, read the draft, and contributed to the final version of the manuscript. Fabrizio Monaco and Nicola Villari managed the patients' sedation and ventilation during the procedures, collected patients' data and informed consent, and reviewed the final version of the manuscript. Carmine D. Votta contributed to data collection and wrote the initial draft of the manuscript. He is also the corresponding author.

Editorial responsibility This submission was handled by Dr. Hilary P. Grocott, Editor-in-Chief, Canadian Journal of Anesthesia.

\section{References}

1. Camm AJ, Lip GY, De Caterina R, et al. 2012 focused update of the ESC Guidelines for the management of atrial fibrillation: an update of the 2010 ESC Guidelines for the management of atrial fibrillation. Developed with the special contribution of the European Heart Rhythm Association. Eur Heart J 2012; 33: 2719-47.

2. Regazzoli D, Ancona F, Trevisi N, et al. Left atrial appendage: physiology, pathology, and role as therapeutic target. Biomed Res Int 2015; 2015: 205013.

3. Murphy GS, Szokol JW, Marymont JH, Greenberg SB, Avram MJ, Vender JS. Residual neuromuscular blockade and critical respiratory events in the postanesthesia care unit. Anesth Analg 2008; 107: 130-7.

4. Aytac I, Postaci A, Aytac B, et al. Survey of postoperative residual curarization, acute respiratory events and approach of anesthesiologists. Braz J Anesthesiol 2016; 66: 55-62.

5. Qiao $Y$, Feng $H$, Zhao T, Yan H, Zhang H, Zhao X. Postoperative cognitive dysfunction after inhalational anesthesia in elderly patients undergoing major surgery: the influence of anesthetic technique, cerebral injury and systemic inflammation. BMC Anesthesiol 2015; 15: 154.

6. Motloch LJ, Rottlaender D, Reda S, et al. Local versus general anesthesia for transfemoral aortic valve implantation. Clin Res Cardiol 2012; 101: 45-53.

7. Chernik DA, Gillings D, Laine $H$, et al. Validity and reliability of the Observer's Assessment of Alertness/Sedation Scale: study with intravenous midazolam. J Clin Psychopharmacol 1990; 10: 244-51.

8. Cabrini $L$, Landoni $G$. A novel non-invasive ventilation mask to prevent and manage respiratory failure during fiberoptic bronchoscopy, gastroscopy and transesophageal echocardiography. Heart Lung Vessel 2015; 7: 297-303.

9. Smetana GW, Lawrence VA. Cornell JE; American College of Physicians. Preoperative pulmonary risk stratification for noncardiothoracic surgery: systematic review for the American College of Physicians. Ann Intern Med 2006; 144: 581-95.

10. Eastwood PR, Platt PR, Shepherd K, Maddison K, Hillman DR. Collapsibility of the upper airway at different concentrations of propofol anesthesia. Anesthesiology 2005; 103: 470-7.

11. Sastre JA, Cordovilla R, Jimenez MF, Lopez T. Management of transbronchial cryobiopsy using the i-gel ${ }^{\circledR}$ airway and the Arndt endobronchial blocker. Can J Anesth 2014; 61: 886-8. 